Original Research Paper

\title{
Effect of Bromocriptine and Nano-Bromocriptine on Egg Quality Parameters of Late Laying Hens
}

\author{
Ahmed Dawod, Noha Osman, Hanim S. Heikal and Hamada Mahboub \\ Department of Husbandry and Animal Wealth Development, Faculty of Veterinary Medicine, \\ University of Sadat City, Menofia, Egypt
}

\author{
Article history \\ Received: 10-06-2021 \\ Revised: 02-09-2021 \\ Accepted: 06-09-2021 \\ Corresponding Author: \\ Ahmed Dawod \\ Department of Husbandry and \\ Animal Wealth Development, \\ Faculty of Veterinary Medicine, \\ University of Sadat City, \\ Menofia, Egypt \\ Email: adawod280@gmail.com
}

\begin{abstract}
The study was conducted to determine the effect of bromocriptine and nano-bromocriptine on external and internal egg qualities of late laying hens. A total of 150 Novo-gene brown strain hens of 70 weeks of age were selected from a commercial laying farm. The birds were divided randomly into three groups, with 50 birds in each. The first group was given saline and kept as control, while the second and third groups were given bromocriptine (2-bromoalpha-ergocriptine) and nano-bromocriptine, respectively. Each group was separated into two subgroups of equal number, the first receiving the treatment orally and the second receiving the treatment via subcutaneous injections at a dose of $100 \mathrm{microg} / \mathrm{kg}$ body weight/ week. During experimentation, five eggs were taken every 4 weeks from each subgroup to determine the internal and the external egg qualities. Results revealed that Administration of either bromocriptine or nano-bromocriptine increased significantly the egg weight, egg length, yolk height, yolk weight, yolk width and thick albumin height. The findings conclude that bromocriptine and nano-bromocriptine treatment could be used for the late laying hens to improve the internal and external qualities of eggs.
\end{abstract}

Keywords: Bromocriptine, Nano-Bromocriptine, Egg Quality, Late Laying Hens

\section{Introduction}

Currently, the problems of egg quality cost the egg industry huge costs (Alwell et al., 2017). Since the consumer acceptance of the eggs is mainly influenced by their quality. Therefore, the production of eggs with good internal and external qualities is a vital subject in the egg industry. The majority of egg quality traits altered mainly as a result of nutrition, environmental conditions, genotype, age of hens and laying rate (Van den Brand et al., 2004). After 60 weeks of age, the laying rate begins to decline due to the large range in clutch length across hens (Thiruvenkadan et al., 2010). This is accompanied by a decline in egg quality including both internal and external qualities, which are resulting in breakage during collection and transportation (Dunn, 2013). As, the hens grew older, the egg weight and size were increased, whereas the eggshell breaking strength and Haugh unit was declined (Chang-Ho et al., 2014).

Many factors influence the functional quality of the eggshell, including the strain, age of the bird, nutrition, stress, disease and the housing system (Vlčková et al., 2018). The eggshell is considered as the natural packing material for the egg content. Thus, higher eggshell strength will be beneficial in securing salable eggs during the production chain. The eggshell's integrity is critical as a natural defense mechanism that protects eggs from physical and microbiological damage. During the last stages of production, the eggshell strength deteriorates (Bar et al., 2002), which could be resulted in a high rate of cracked eggs as it may be reached to $20 \%$ during such stage (Nys, 2001).

Prolactin is one of the adenohypophysis hormones. This hormone is considered as one of the much-blamed factors concerning with decreasing egg-laying performance especially during the last stage of laying. Prolactin was tremendously increased with the progression of laying and reached its high level during late laying (Reddy et al., 2001). Many research trials tried to overcome the high level of prolactin during such stage of production via using bromocriptine. Bromocriptine is an ergot derivative that has strong dopamine agonistic effect. This drug is a semi-synthetic derivative of ergocryptine that stimulates the pituitary's Dopaminergic receptors (D2). It stops the anterior lobe of the pituitary gland from producing and secreting prolactin. Moreover, it slows the progression of pituitary adenoma (prolactinoma) and decreases the level of prolactin in hyperprolactinemia (Molik 
and Blasiak, 2015). Also, bromocriptine is used to treat acromegaly since it inhibits the release of growth hormone (Keche, 2010).

Recently, attention has been drawn toward liquid self-nano emulsifying drug delivery systems (Hussein and Rajab, 2018). Since the rate and extent of release for all nanoform prepared formulations were significantly higher than conventional drugs. Therefore, self-nano emulsifying drug delivery system is a promising approach to improve solubility, dissolution and stability of the formulation (Hussein and Rajab, 2018). For enhanced absorption and therapeutic efficacy via the oro-trans labio mucosal route, nanosized bromocriptine-loaded mucoadhesive bio-flexy films were created utilizing biopolymer from Zea mays. The unique formulation technique for bromocriptine delivery may serve as a landmark for Parkinson's treatment and management due to the benefits of single daily dosing and reduced dose-related side effects (Jaiswal and Madhav, 2019). Moreover, bromocriptine alginate nanocomposite was prepared to maximize the half-life, stability, solubility and permeability of bromocriptine (Siddique et al., 2016). The current study aimed to improve egg internal and external qualities during the late laying stage via administration of a minimal amount of either bromocriptine or nano-bromocriptine (bromocriptine alginate nanocomposite) for late laying hens.

\section{Materials and Methods}

\section{Ethical Statement}

The study was approved by the Institutional Animal Care and Use Committee (IACUC), Faculty of Veterinary Medicine, University of Sadat City, Egypt (Ethical approval number: VUSC-017-1-19)

\section{Preparation of Nano-Bromocriptine}

The nano-bromocriptine was prepared as an alginate-bromocriptine nano-composite according to (Siddique et al., 2016). Concisely, $100 \mathrm{mg}$ of 2-Bromo- $\alpha-$ ergocryptine (Sigma, USA) was liquefied in $5 \mathrm{~mL}$ ethanol then added wisely to sodium alginate solution. The sodium alginate solution was prepared via dissolving of $0.1 \mathrm{gm}$ of sodium alginate (Fisher Scientific, UK) in $100 \mathrm{~mL}$ distilled water with stirring. The adding procedure is done drop by drop while mixing with careful stirring and worming for $30 \mathrm{~min}$. Then the solution was ultra-sonicated at $50^{\circ} \mathrm{C}$ and $100 \mathrm{w}$ with $35 \mathrm{kHz}$ for $45 \mathrm{~min}$. Finally, the prepared nanocomposite solution was air-dried and kept in a cool and dry environment.

\section{Animals and Experimental Design}

A total of 150 Novo-gene brown strain 70-week-old hens were chosen from a commercial farm. The chickens were selected based on the quality of their feathers, body weight and lack of deformities. The average bodyweight of the selected laying hens was $1800 \pm 150 \mathrm{gm}$. The birds were split into three groups, each with 50 birds then the birds were subjected to 2 weeks acclimatization period. The first group was given saline and kept as a placebo, while the second and third groups were given bromocriptine (2-bromo-alphaergocriptine, Sigma, USA) and nano-bromocriptine, respectively. Each group was split into two subgroups of equal size, the first take the treatment orally while the second take it subcutaneously underneath the wing. The doses of bromocriptine and nano-bromocriptine were adjusted to $100 \mathrm{microg} / \mathrm{kg}$ body weight/week (Reddy et al., 2007). The birds take the treatments from the beginning of the $72^{\text {nd }}$ till the end of the $84^{\text {th }}$ week of age. A commercial laying pelleted ration with 16 percent crude protein (corn-soybean meal with $16 \% \mathrm{CP} ; 2700$ $\mathrm{kcal} \mathrm{ME} / \mathrm{kg}$ diet) and ad libitum freshwater were fed to the hens daily.

\section{Measurements}

During experimentation, five eggs were taken randomly at monthly intervals from each subgroup for the determination of different egg quality parameters. A variety of quality parameters were used to assess either external or internal egg quality including egg weight, egg length, egg width, egg shell weight including inner shell membrane, eggshell thickness, albumen weight, thick albumen height, thick albumin width, Yolk width, Yolk height and Yolk weight.

Thick albumin width was determined as the distance between the two far points in thick albumin while the egg was broken in the Petri dish of $20 \mathrm{~cm}$ diameter. Also, the albumin height was determined from various points of albumin and the mean of these values was determined as albumin height. Moreover, the yolk width was determined as the width between the two poles of the yolk at the level of the two chalazae.

An electronic balance was used to measure the weights of the various egg components (total egg, yolk, albumin and eggshell weights) with an accuracy of $0.1 \mathrm{gm}$. Moreover, before weighing the eggshell, the adhering albumen was removed using a paper towel. Then the weight of albumin was estimated by subtracting the yolk and shell weight from the total weight of the egg. A manual caliper was used to measure the length, width of the eggs, albumin, yolk and eggshell thickness with an accuracy of $0.01 \mathrm{~cm}$. Furthermore, the eggshell thickness was determined at 3 different sections in the dry eggshell with intact inner shell membrane around the equator of the egg (Molnár et al., 2016). The length of the egg was measured from pole to pole, while the width was measured at the equator (Coucke et al., 1999).

\section{Statistical Analysis}

After the conduction of the experiment, the data were enrolled into two-way ANOVA using the General Linear Models (GLM) procedures of SAS software (SAS User's 
Guide: Statistics, Version 8.1 Edition, 2000, SAS Inst. Inc., Cary, NC) according to the following statistical model:

$$
Y_{i j k}=\mu+\alpha_{i}+\beta_{j}+(\alpha \beta)_{i j}+\epsilon_{i j k}
$$

where, $Y_{i j k}=$ overall observation, $\mu=$ overall mean, $\alpha_{i}$ was the treatment effect $i=1,2,3$ for control, bromocriptine and nano-bromocriptine treatments, $\beta_{j}$ was the route of administration $j=1,2$ for oral and injection rout of administrations, $(\alpha \beta)_{i j}$ was the interaction between treatments and route of administration and $\epsilon_{i j k}$ was the random error. Tukey test was used as mean separation test and results were expressed as least square means $\pm \mathrm{SE}$. The level of significance was seated at $(\mathrm{P}<0.05)$.

\section{Results}

\section{Effect of Bromocriptine and Nano-bromocriptine on external egg quality}

Administration of bromocriptine significantly increases egg weight $(63.83 \pm 1.16 \mathrm{gm})$ than the control group $(59.90 \pm 1.17 \mathrm{gm})$ at $84^{\text {th }}$ week age (Table 1$)$. Where the administration of nano-bromocriptine sustained intermediate egg weight value $(60.92 \pm 1.08 \mathrm{gm})$ between the bromocriptine and control groups $(\mathrm{P}<0.05)$. In contrast, neither bromocriptine nor nano-bromocriptine administration to the laying hens possessed any significant effect on egg weight during the $76^{\text {th }}$ or $80^{\text {th }}$ week of age. Moreover, bromocriptine treatment birds increased significantly the egg length $(58.91 \pm 0.45 \mathrm{~mm})$ rather than nano-bromocriptine and control-treated ones $(57.45 \pm 0.42 ; 57.32 \pm 0.45)$ at $84^{\text {th }}$ week of age.
Concerning the egg width (Table 2), it was clear that egg width possessed no significant differences among different experimental groups. However, the eggshell weight at the $80^{\text {th }}$ week of age was significantly increased in the bromocriptine treated group $(7.10 \pm 0.25 \mathrm{gm})$ followed by the nano-bromocriptine treated group $(6.40 \pm 0.16 \mathrm{gm})$ and control $(6.28 \pm 0.20 \mathrm{gm})(P<0.0276)$. While at $84^{\text {th }}$ week age there was no significant difference in eggshell weight between different groups. Moreover, either bromocriptine or nano-bromocriptine treatment had no significant effect on eggshell thickness.

\section{Effect of Bromocriptine and Nano-bromocriptine on Internal Egg Quality}

Regarding the effect of the bromocriptine and nanobromocriptine on the albumin weight (Table 3), it was evident that bromocriptine and nano-bromocriptine administration had no significant effect on albumin weight. In contraries, thick albumin height was significantly higher in control $(6.99 \pm 0.18 \mathrm{~mm})$ than nano-bromocriptine treated group $(6.13 \pm 0.16 \mathrm{~mm})$ which was not significantly differed from bromocriptine treated group $(6.56 \pm 0.17 \mathrm{~mm})$ at $84^{\text {th }}$ week age $(\mathrm{P}<0.0038)$ (Table 4$)(P<0.0038)$. While control (orally and injection) and bromocriptine injection groups were of significantly higher thick albumin height than other groups. Also, oral nano-bromocriptine improved significantly the thick albumen height $(6.43 \pm 0.21 \mathrm{~mm})$ than injectable nano-bromocriptine $(5.83 \pm 0.25 \mathrm{~mm})$. Moreover, bromocriptine and nano-bromocriptine administration increased significantly the thick albumin width at $76^{\text {th }}$ $(102.56 \pm 2.47 ; \quad 101.77 \pm 1.51 \mathrm{~mm})$ and $84^{\text {th }}$ $(101.85 \pm 1.68 ; 106.21 \pm 1.57 \mathrm{~mm})$ week of age than the control group. Also, oral bromocriptine and injectable nano- bromocriptine had the highest thick albumin width $(107.20 \pm 2.58 ; 109.75 \pm 2.39 \mathrm{~mm})$ compared with other groups at $84^{\text {th }}$ week of age $(\mathrm{P}<0.0001)$.

Table 1: Effects of bromocriptine and nano-bromocriptine on egg weight and length of late laying hens

\begin{tabular}{|c|c|c|c|c|c|c|c|}
\hline \multirow[t]{2}{*}{ Parameter } & \multicolumn{3}{|l|}{ Egg weight $(\mathrm{gm})$} & \multicolumn{4}{|c|}{ Egg length $(\mathrm{mm})$} \\
\hline & $76^{\text {th }}$ week & $80^{\text {th }}$ week & $84^{\text {th }}$ week & $76^{\text {th }}$ week & $80^{\text {th }}$ week & $84^{\text {th }}$ week & \\
\hline \multirow[t]{3}{*}{ Treatment } & Control & $63.80 \pm 2.09$ & $61.55 \pm 1.60$ & $59.90 \pm 1.17^{\mathrm{b}}$ & $58.41 \pm 0.38$ & $57.59 \pm 0.92$ & $57.32 \pm 0.45^{\mathrm{b}}$ \\
\hline & Bromocriptine & $70.57 \pm 2.21$ & $65.91 \pm 2.13$ & $63.83 \pm 1.16^{\mathrm{a}}$ & $60.37 \pm 0.88$ & $59.17 \pm 0.88$ & $58.91 \pm 0.45^{\mathrm{a}}$ \\
\hline & Nano-bromocriptine & $70.86 \pm 2.06$ & $63.42 \pm 1.75$ & $60.92 \pm 1.08^{\mathrm{ab}}$ & $61.29 \pm 0.88$ & $59.21 \pm 0.49$ & $57.45 \pm 0.42^{\mathrm{b}}$ \\
\hline \multirow[t]{2}{*}{ Administration } & Orally & $68.99 \pm 1.94$ & $63.76 \pm 1.73$ & $61.74 \pm 0.92$ & $60.18 \pm 0.75$ & $58.73 \pm 0.67$ & $58.10 \pm 0.35$ \\
\hline & Injection & $67.84 \pm 1.86$ & $63.50 \pm 1.34$ & $61.36 \pm 0.93$ & $59.86 \pm 0.62$ & $58.59 \pm 0.63$ & $57.68 \pm 0.36$ \\
\hline \multirow{7}{*}{$\begin{array}{l}\text { Treatment } \chi \\
\text { administration }\end{array}$} & & & & & & & \\
\hline & Control (oral) & $64.58 \pm 2.99$ & $61.88 \pm 2.19$ & $59.95 \pm 1.59$ & $58.41 \pm 0.51$ & $57.68 \pm 1.24$ & $57.32 \pm 0.61$ \\
\hline & Control (injection) & $63.03 \pm 3.30$ & $61.23 \pm 2.70$ & $59.85 \pm 1.71$ & $58.40 \pm 0.66$ & $57.51 \pm 1.59$ & $57.32 \pm 0.65$ \\
\hline & Bromocriptine (oral) & $71.64 \pm 3.14$ & $65.70 \pm 3.61$ & $64.74 \pm 1.78$ & $60.94 \pm 1.33$ & $59.41 \pm 1.36$ & $59.53 \pm 0.68$ \\
\hline & Bromocriptine (injection) & $69.50 \pm 3.39$ & $66.12 \pm 2.69$ & $62.92 \pm 1.49$ & $59.79 \pm 1.25$ & $58.93 \pm 1.26$ & $58.29 \pm 0.57$ \\
\hline & Nano-bromocriptine (oral) & $70.74 \pm 3.62$ & $63.70 \pm 3.42$ & $60.54 \pm 1.41$ & $61.19 \pm 1.66$ & $59.09 \pm 0.98$ & $57.46 \pm 0.54$ \\
\hline & Nano-bromocriptine (injection) & $70.98 \pm 2.42$ & $63.14 \pm 1.41$ & $61.31 \pm 1.65$ & $61.40 \pm 0.83$ & $59.34 \pm 0.34$ & $57.44 \pm 0.63$ \\
\hline \multirow[t]{3}{*}{$\mathrm{P}$-value } & Treatment & 0.0678 & 0.3175 & 0.0500 & 0.6032 & 0.3218 & 0.0216 \\
\hline & Administration & 0.6616 & 0.9086 & 0.7702 & 0.7361 & 0.8884 & 0.4058 \\
\hline & Interaction & 0.9246 & 0.9773 & 0.7135 & 0.8135 & 0.9511 & 0.5215 \\
\hline
\end{tabular}

gm: Gram; mm: Millimeter. Values are presented as least square mean \pm SE. ${ }^{a-d}$ Means within the same column for each parameter with different superscripts are statistically different at $P<0.05$ (Two way ANOVA, Tukey post- hoc test) 
Ahmed Dawod et al. / American Journal of Pharmacology and Toxicology 2021, Volume 16: 17.24 DOI: 10.3844/ajptsp.2021.17.24

Table 2: Effects of bromocriptine and nano-bromocriptine on egg width and eggshell weight of late laying hens

\begin{tabular}{|c|c|c|c|c|c|c|c|}
\hline \multicolumn{2}{|l|}{ Parameter } & \multicolumn{3}{|c|}{ Egg width $(\mathrm{mm})$} & \multicolumn{3}{|c|}{ Eggshell weight (gm) } \\
\hline & & \multirow{2}{*}{$\frac{76^{\text {th }} \text { week }}{44.12 \pm 0.52}$} & \multirow{2}{*}{$\frac{80^{\text {th }} \text { week }}{43.77 \pm 0.28}$} & \multirow{2}{*}{$\frac{84^{\text {th }} \text { week }}{43.25 \pm 0.29}$} & \multirow{2}{*}{$\frac{76^{\text {th }} \text { week }}{6.79 \pm 0.36}$} & \multirow{2}{*}{$\frac{80^{\text {th }} \text { week }}{6.28 \pm 0.20^{\mathrm{b}}}$} & \multirow{2}{*}{$\frac{84^{\text {th }} \text { week }}{5.99 \pm 0.17}$} \\
\hline Treatment & Control & & & & & & \\
\hline & Bromocriptine & $45.77 \pm 0.52$ & $44.68 \pm 0.54$ & $44.18 \pm 0.29$ & $7.43 \pm 0.17$ & $7.10 \pm 0.25^{\mathrm{a}}$ & $6.44 \pm 0.17$ \\
\hline & Nano-bromocriptine & $45.61 \pm 0.46$ & $43.99 \pm 0.45$ & $43.71 \pm 0.27$ & $7.07 \pm 0.21$ & $6.40 \pm 0.16^{b}$ & $5.96 \pm 0.16$ \\
\hline \multirow[t]{2}{*}{ Administration } & Orally & $45.30 \pm 0.45$ & $44.20 \pm 0.40$ & $43.77 \pm 0.23$ & $7.21 \pm 0.23$ & $6.51 \pm 0.21$ & $6.03 \pm 0.14$ \\
\hline & Injection & $45.04 \pm 0.44$ & $44.09 \pm 0.35$ & $43.65 \pm 0.24$ & $6.99 \pm 0.19$ & $6.67 \pm 0.17$ & $6.23 \pm 0.14$ \\
\hline \multirow{6}{*}{$\begin{array}{l}\text { Treatment } \chi \\
\text { administration }\end{array}$} & Control (oral) & $44.44 \pm 0.82$ & $43.84 \pm 0.39$ & $43.27 \pm 0.40$ & $6.76 \pm 0.49$ & $6.36 \pm 0.29$ & $5.95 \pm 0.24$ \\
\hline & Control (injection) & $43.80 \pm 0.65$ & $43.69 \pm 0.47$ & $43.22 \pm 0.43$ & $6.83 \pm 0.62$ & $6.20 \pm 0.31$ & $6.03 \pm 0.26$ \\
\hline & Bromocriptine (oral) & $45.82 \pm 0.72$ & $44.64 \pm 0.85$ & $44.42 \pm 0.45$ & $7.70 \pm 0.20$ & $6.94 \pm 0.48$ & $6.37 \pm 0.27$ \\
\hline & Bromocriptine (injection) & $45.73 \pm 0.84$ & $44.71 \pm 0.77$ & $43.94 \pm 0.38$ & $7.16 \pm 0.22$ & $7.26 \pm 0.21$ & $6.52 \pm 0.22$ \\
\hline & Nano-bromocriptine (oral) & $45.64 \pm 0.80$ & $44.12 \pm 0.83$ & $43.61 \pm 0.36$ & $7.16 \pm 0.42$ & $6.24 \pm 0.27$ & $5.77 \pm 0.21$ \\
\hline & Nano-bromocriptine (injection) & $45.58 \pm 0.57$ & $43.86 \pm 0.48$ & $43.80 \pm 0.41$ & $6.98 \pm 0.13$ & $6.56 \pm 0.17$ & $6.15 \pm 0.25$ \\
\hline \multirow[t]{3}{*}{ P-value } & Treatment & 0.0788 & 0.3851 & 0.0863 & 0.2478 & 0.0276 & 0.0898 \\
\hline & Administration & 0.6702 & 0.83341 & 0.7338 & 0.4757 & 0.5295 & 0.3026 \\
\hline & Interaction & 0.9133 & 0.9684 & 0.6954 & 0.7174 & 0.6802 & 0.8102 \\
\hline
\end{tabular}

gm: Gram; mm: Millimeter. Values are presented as least square mean \pm SE. ${ }^{\text {a-d }}$ Means within the same column for each parameter with different superscripts are statistically different at $P<0.05$ (Two way ANOVA, Tukey post- hoc test)

Table 3: Effects of bromocriptine and nano-bromocriptine on eggshell thickness and albumin weight of late laying hens

\begin{tabular}{|c|c|c|c|c|c|c|c|}
\hline \multirow{2}{*}{ Parameter } & & \multicolumn{3}{|c|}{ Eggshell thickness (mm) } & \multicolumn{3}{|c|}{ Albumin weight (gm) } \\
\hline & & $76^{\text {th }}$ week & $80^{\text {th }}$ week & $84^{\text {th }}$ week & $76^{\text {th }}$ week & $80^{\text {th }}$ week & $84^{\text {th }}$ week \\
\hline \multirow[t]{3}{*}{ Treatment } & Control & $0.38 \pm 0.01$ & $0.37 \pm 0.00$ & $0.37 \pm 0.01$ & $40.88 \pm 1.44$ & $40.84 \pm 1.27$ & $39.44 \pm 0.94$ \\
\hline & Bromocriptine & $0.38 \pm 0.01$ & $0.39 \pm 0.01$ & $0.37 \pm 0.01$ & $46.70 \pm 1.84$ & $42.63 \pm 1.74$ & $42.17 \pm 0.93$ \\
\hline & Nano-bromocriptine & $0.37 \pm 0.01$ & $0.37 \pm 0.01$ & $0.37 \pm 0.00$ & $46.71 \pm 1.72$ & $41.58 \pm 1.56$ & $40.04 \pm 0.87$ \\
\hline \multirow[t]{2}{*}{ Administration } & Orally & $0.38 \pm 0.00$ & $0.38 \pm 0.01$ & $0.36 \pm 0.00$ & $45.13 \pm 1.59$ & $42.07 \pm 1.39$ & $40.75 \pm 0.74$ \\
\hline & Injection & $0.38 \pm 0.01$ & $0.38 \pm 0.01$ & $0.37 \pm 0.00$ & $44.39 \pm 1.47$ & $41.30 \pm 1.08$ & $40.35 \pm 0.75$ \\
\hline \multirow{6}{*}{$\begin{array}{l}\text { Treatment } \chi \\
\text { administration }\end{array}$} & Control (oral) & $0.38 \pm 0.01$ & $0.38 \pm 0.01$ & $0.37 \pm 0.01$ & $41.56 \pm 2.14$ & $41.16 \pm 1.76$ & $39.55 \pm 1.28$ \\
\hline & Control (injection) & $0.38 \pm 0.01$ & $0.37 \pm 0.01$ & $0.37 \pm 0.01$ & $40.20 \pm 2.13$ & $40.53 \pm 2.12$ & $39.33 \pm 1.37$ \\
\hline & Bromocriptine (oral) & $0.38 \pm 0.01$ & $0.38 \pm 0.01$ & $0.36 \pm 0.01$ & $47.28 \pm 2.67$ & $43.16 \pm 2.76$ & $42.82 \pm 1.43$ \\
\hline & Bromocriptine (injection) & $0.38 \pm 0.01$ & $0.39 \pm 0.02$ & $0.37 \pm 0.01$ & $46.12 \pm 2.81$ & $42.10 \pm 2.42$ & $41.52 \pm 1.20$ \\
\hline & Nano-bromocriptine (oral) & $0.37 \pm 0.01$ & $0.37 \pm 0.01$ & $0.36 \pm 0.01$ & $46.56 \pm 3.15$ & $41.90 \pm 3.01$ & $39.88 \pm 1.13$ \\
\hline & Nano-bromocriptine (injection) & $0.37 \pm 0.01$ & $0.37 \pm 0.01$ & $0.37 \pm 0.01$ & $46.86 \pm 1.83$ & $41.26 \pm 1.35$ & $40.21 \pm 1.32$ \\
\hline \multirow[t]{3}{*}{ P-value } & Treatment & 0.4609 & 0.2448 & 0.7826 & 0.0517 & 0.7494 & 0.0991 \\
\hline & Administration & 0.9824 & 0.6842 & 0.1307 & 0.7242 & 0.6872 & 0.7086 \\
\hline & Interaction & 0.9225 & 0.8464 & 0.6714 & 0.9376 & 0.9945 & 0.8144 \\
\hline
\end{tabular}

gm: gram; mm: millimeter. Values are presented as least square mean \pm SE. ${ }^{\text {a-d }}$ Means within the same column for each parameter with different superscripts are statistically different at $P<0.05$ (Two way ANOVA, Tukey post- hoc test)

Table 4: Effects of bromocriptine and nano-bromocriptine on thick albumen height and width of late laying hens

\begin{tabular}{|c|c|c|c|c|c|c|c|}
\hline \multirow{2}{*}{ Parameter } & & \multicolumn{3}{|c|}{ Thick albumen height (mm) } & \multicolumn{3}{|c|}{ Thick albumin width (mm) } \\
\hline & & $76^{\text {th }}$ week & $80^{\text {th }}$ week & $84^{\text {th }}$ week & $76^{\text {th }}$ week & $80^{\text {th }}$ week & $84^{\text {th }}$ week \\
\hline \multirow[t]{3}{*}{ Treatment } & Control & $6.18 \pm 0.14$ & $6.14 \pm 0.22$ & $6.99 \pm 0.18^{\mathrm{a}}$ & $87.53 \pm 1.47^{b}$ & $100.35 \pm 2.50$ & $89.92 \pm 1.69^{b}$ \\
\hline & Bromocriptine & $6.38 \pm 0.29$ & $6.38 \pm 0.26$ & $6.56 \pm 0.17^{\mathrm{ab}}$ & $102.56 \pm 2.47^{\mathrm{a}}$ & $102.79 \pm 2.72$ & $101.85 \pm 1.68^{\mathrm{a}}$ \\
\hline & Nano-bromocriptine & $6.47 \pm 0.24$ & $5.98 \pm 0.16$ & $6.13 \pm 0.16^{\mathrm{b}}$ & $101.77 \pm 1.51^{\mathrm{a}}$ & $106.93 \pm 2.43$ & $106.21 \pm 1.57^{\mathrm{a}}$ \\
\hline \multirow[t]{2}{*}{ Administration } & Orally & $6.21 \pm 0.11$ & $6.20 \pm 0.18$ & $6.51 \pm 0.14$ & $97.92 \pm 2.10$ & $103.74 \pm 2.19$ & $99.84 \pm 1.34$ \\
\hline & Injection & $6.48 \pm 0.25$ & $6.14 \pm 0.18$ & $6.61 \pm 0.14$ & $96.66 \pm 2.67$ & $102.98 \pm 2.17$ & $98.82 \pm 1.36$ \\
\hline \multirow{6}{*}{$\begin{array}{l}\text { Treatment } \chi \\
\text { administration }\end{array}$} & Control (oral) & $6.24 \pm 0.20$ & $6.30 \pm 0.37$ & $7.03 \pm 0.24^{\mathrm{a}}$ & $88.33 \pm 2.24$ & $99.14 \pm 3.73$ & $89.66 \pm 2.31^{\mathrm{d}}$ \\
\hline & Control (injection) & $6.13 \pm 0.21$ & $5.98 \pm 0.23$ & $6.94 \pm 0.26^{\mathrm{a}}$ & $86.73 \pm 2.02$ & $101.57 \pm 3.65$ & $90.18 \pm 2.48^{\mathrm{cd}}$ \\
\hline & Bromocriptine (oral) & $6.24 \pm 0.20$ & $6.15 \pm 0.35$ & $6.05 \pm 0.27^{\mathrm{bc}}$ & $102.75 \pm 1.93$ & $103.21 \pm 4.22$ & $107.20 \pm 2.58^{\mathrm{ab}}$ \\
\hline & Bromocriptine (injection) & $6.52 \pm 0.57$ & $6.61 \pm 0.39$ & $7.07 \pm 0.22^{\mathrm{a}}$ & $102.37 \pm 4.88$ & $102.37 \pm 3.92$ & $96.51 \pm 2.17^{\mathrm{c}}$ \\
\hline & Nano-bromocriptine (oral) & $6.15 \pm 0.20$ & $6.13 \pm 0.27$ & $6.43 \pm 0.21^{\mathrm{b}}$ & $102.67 \pm 1.74$ & $108.86 \pm 2.57$ & $102.67 \pm 2.05^{\mathrm{b}}$ \\
\hline & Nano-bromocriptine (injection) & $6.79 \pm 0.41$ & $5.82 \pm 0.18$ & $5.83 \pm 0.25^{\mathrm{c}}$ & $100.87 \pm 2.62$ & $104.99 \pm 4.26$ & $109.75 \pm 2.39^{\mathrm{a}}$ \\
\hline \multirow[t]{3}{*}{ P-value } & Treatment & 0.7020 & 0.4274 & 0.0038 & $<.0001$ & 0.2409 & $<.0001$ \\
\hline & Administration & 0.3354 & 0.8193 & 0.5846 & 0.5930 & 0.8083 & 0.5922 \\
\hline & Interaction & 0.5554 & 0.3683 & 0.0040 & 0.9635 & 0.7182 & 0.0010 \\
\hline
\end{tabular}

gm: Gram; mm: Millimeter. Values are presented as least square mean \pm SE. ${ }^{a-d}$ Means within the same column for each parameter with different superscripts are statistically different at $P<0.05$ (Two way ANOVA, Tukey post- hoc test) 
Table 5: Effects of bromocriptine and nano-bromocriptine on yolk weight and width of late laying hens

\begin{tabular}{|c|c|c|c|c|c|c|c|}
\hline \multirow[t]{2}{*}{ Parameter } & & \multicolumn{2}{|c|}{ Yolk weight (gm) } & \multirow[b]{2}{*}{$84^{\text {th }}$ week } & \multirow[b]{2}{*}{$76^{\text {th }}$ week } & \multicolumn{2}{|c|}{ Yolk width (mm) } \\
\hline & & $76^{\text {th }}$ week & $80^{\text {th }}$ week & & & $80^{\text {th }}$ week & $84^{\text {th }}$ week \\
\hline \multirow[t]{3}{*}{ Treatment } & Control & $15.32 \pm 0.41$ & $14.00 \pm 0.28^{b}$ & $14.24 \pm 0.26$ & $39.35 \pm 0.25$ & $39.06 \pm 0.35^{\mathrm{b}}$ & $37.86 \pm 0.27^{b}$ \\
\hline & Bromocriptine & $16.08 \pm 0.49$ & $15.81 \pm 0.39^{\mathrm{a}}$ & $14.98 \pm 0.26$ & $39.70 \pm 0.40$ & $40.05 \pm 0.20^{\mathrm{a}}$ & $38.27 \pm 0.27^{\mathrm{ab}}$ \\
\hline & Nano-bromocriptine & $16.71 \pm 0.44$ & $15.22 \pm 0.28^{\mathrm{a}}$ & $14.67 \pm 0.25$ & $40.72 \pm 0.59$ & $40.11 \pm 0.27^{\mathrm{a}}$ & $38.80 \pm 0.25^{\mathrm{a}}$ \\
\hline \multirow[t]{2}{*}{ Administration } & Orally & $16.12 \pm 0.35$ & $14.85 \pm 0.30$ & $14.70 \pm 0.21$ & $40.17 \pm 0.37$ & $39.72 \pm 0.30$ & $38.08 \pm 0.21$ \\
\hline & Injection & $15.95 \pm 0.43$ & $15.17 \pm 0.36$ & $14.56 \pm 0.21$ & $39.68 \pm 0.41$ & $39.76 \pm 0.20$ & $38.54 \pm 0.21$ \\
\hline \multirow{6}{*}{$\begin{array}{l}\text { Treatment } \chi \\
\text { administration }\end{array}$} & Control (oral) & $15.46 \pm 0.58$ & $13.92 \pm 0.39$ & $14.22 \pm 0.36$ & $39.51 \pm 0.40$ & $38.81 \pm 0.57$ & $37.90 \pm 0.37$ \\
\hline & Control (injection) & $15.18 \pm 0.65$ & $14.08 \pm 0.46$ & $14.25 \pm 0.39$ & $39.18 \pm 0.28$ & $39.31 \pm 0.37$ & $37.83 \pm 0.39$ \\
\hline & Bromocriptine (oral) & $16.30 \pm 0.74$ & $15.28 \pm 0.62$ & $15.28 \pm 0.40$ & $40.08 \pm 0.59$ & $39.91 \pm 0.22$ & $38.30 \pm 0.41$ \\
\hline & Bromocriptine (injection) & $15.86 \pm 0.72$ & $16.34 \pm 0.42$ & $14.68 \pm 0.34$ & $39.32 \pm 0.53$ & $40.19 \pm 0.35$ & $38.25 \pm 0.34$ \\
\hline & Nano-bromocriptine (oral) & $16.60 \pm 0.50$ & $15.34 \pm 0.26$ & $14.61 \pm 0.32$ & $40.91 \pm 0.81$ & $40.44 \pm 0.44$ & $38.05 \pm 0.32$ \\
\hline & Nano-bromocriptine (injection) & $16.82 \pm 0.77$ & $15.10 \pm 0.53$ & $14.74 \pm 0.37$ & $40.53 \pm 0.95$ & $39.78 \pm 0.28$ & $39.54 \pm 0.38$ \\
\hline \multirow[t]{3}{*}{$\mathrm{P}$-value } & Treatment & 0.1459 & 0.0026 & 0.1403 & 0.1201 & 0.0262 & 0.0424 \\
\hline & Administration & 0.7623 & 0.3967 & 0.6170 & 0.3701 & 0.9026 & 0.1369 \\
\hline & Interaction & 0.8737 & 0.3545 & 0.5539 & 0.9384 & 0.3096 & 0.0529 \\
\hline
\end{tabular}

gm: Gram; mm: Millimeter. Values are presented as least square mean \pm SE. ${ }^{\text {a-d }}$ Means within the same column for each parameter with different superscripts are statistically different at $P<0.05$ (Two way ANOVA, Tukey post- hoc test)

Table 6: Effects of bromocriptine and nano-bromocriptine on yolk height of late laying hens

\begin{tabular}{|c|c|c|c|c|}
\hline \multicolumn{2}{|l|}{ Parameter } & \multicolumn{3}{|c|}{ Yolk height (mm) } \\
\hline & & $76^{\text {th }}$ week & $80^{\text {th }}$ week & $84^{\text {th }}$ week \\
\hline \multirow[t]{3}{*}{ Treatment } & Control & $14.63 \pm 0.30$ & $13.31 \pm 0.28^{b}$ & $13.72 \pm 0.14^{\mathrm{a}}$ \\
\hline & Bromocriptine & $14.42 \pm 0.21$ & $14.05 \pm 0.24^{\mathrm{a}}$ & $13.78 \pm 0.14^{\mathrm{a}}$ \\
\hline & Nano-bromocriptine & $14.65 \pm 0.22$ & $13.03 \pm 0.20^{b}$ & $13.24 \pm 0.13^{b}$ \\
\hline \multirow[t]{2}{*}{ Administration } & Orally & $14.62 \pm 0.20$ & $13.40 \pm 0.21$ & $13.64 \pm 0.11$ \\
\hline & Injection & $14.52 \pm 0.19$ & $13.53 \pm 0.24$ & $13.51 \pm 0.11$ \\
\hline \multirow[t]{6}{*}{ Treatment $\chi$ administration } & Control (oral) & $14.85 \pm 0.49$ & $13.36 \pm 0.39$ & $13.68 \pm 0.19$ \\
\hline & Control (injection) & $14.42 \pm 0.29$ & $13.25 \pm 0.48$ & $13.77 \pm 0.20$ \\
\hline & Bromocriptine (oral) & $14.63 \pm 0.21$ & $13.86 \pm 0.41$ & $13.88 \pm 0.21$ \\
\hline & Bromocriptine (injection) & $14.21 \pm 0.37$ & $14.24 \pm 0.27$ & $13.67 \pm 0.17$ \\
\hline & Nano-bromocriptine (oral) & $14.38 \pm 0.33$ & $12.97 \pm 0.22$ & $13.37 \pm 0.17$ \\
\hline & Nano-bromocriptine (injection) & $14.92 \pm 0.27$ & $13.09 \pm 0.36$ & $13.10 \pm 0.19$ \\
\hline \multirow[t]{3}{*}{ P-value } & Treatment & 0.7591 & 0.0215 & 0.0070 \\
\hline & Administration & 0.7137 & 0.6656 & 0.3992 \\
\hline & Interaction & 0.2822 & 0.7930 & 0.6013 \\
\hline
\end{tabular}

mm: Millimeter. Values are presented as least square mean \pm SE. ${ }^{\text {a-d }}$ Means within the same column for each parameter with different superscripts are statistically different at $P<0.05$ (Two way ANOVA, Tukey post- hoc test)

Bromocriptine and nano-bromocriptine administration at $80^{\text {th }}$ week of age increased significantly the yolk weight $(15.81 \pm 0.39$ and $15.22 \pm 0.28 \mathrm{gm}$, respectively) than the control group $(14.00 \pm 0.28 \mathrm{gm})(\mathrm{P}<0.0026)$ (Table 5). Moreover, this effect disappeared at the $84^{\text {th }}$ week of age. Furthermore, both bromocriptine and nano-bromocriptine administration increased significantly the yolk width at $80^{\text {th }}$ week of age $(40.05 \pm 0.20$ and $40.11 \pm 0.27 \mathrm{~mm})$ than the control group $(39.06 \pm 0.35 \mathrm{~mm}) \quad(\mathrm{P}<0.0262)$. Conversely, at the $84^{\text {th }}$ week of age, the yolk width was significantly increased in the nano-bromocriptine group $(38.80 \pm 0.25 \mathrm{~mm})$ than in the control $(37.86 \pm 0.27 \mathrm{~mm})$ $(\mathrm{P}<0.0424)$. The yolk height was significantly increased in the bromocriptine treated group $(14.05 \pm 0.24 \mathrm{~mm})$ than control $(13.31 \pm 0.28 \mathrm{~mm})$ and nano-bromocriptine $(13.03 \pm 0.20 \mathrm{~mm})$ treated groups at the $80^{\text {th }}$ week of age $(\mathrm{P}<0.0215)$ (Table 6$)$. However, at the $84^{\text {th }}$ week of age, the yolk height was significantly increased in control and bromocriptine treated birds $(13.72 \pm 0.14 ; 13.78 \pm 0.14 \mathrm{~mm})$ compared with nano-bromocriptine treated ones $(13.24 \pm 0.13 \mathrm{~mm})(P<0.0070)$.

\section{Discussion}

The findings revealed that both bromocriptine and nano-bromocriptine increased significantly the egg weight, egg length, thick albumen height and width, while it possessed no significant differences in albumin weight. The results could be due to efficient oviduct function sustained via low prolactin resulted from bromocriptine and nano-bromocriptine treatments. Prolactin is considered as one of the negative regulators for reproduction in avian species, as it can suppress gonadotropins and induce ovarian follicles atresia 
(Chaiseha and El Halawani, 2005). Likewise, Parvez et al. (2017) and Banu et al. (2016) showed that there was no significant difference in thick albumin weight or albumin \% between the bromocriptine treated and control group. Moreover, Parvez et al. (2017) showed that the bromocriptine treated group was of significantly lower albumin diameter than the control group. Furthermore, the bromocriptine treatment decreases significantly egg weight in laying hens. However, Banu et al. (2016) reported that there was no significant difference in egg weight between the bromocriptine treated groups and the control group.

Either bromocriptine or nano-bromocriptine treatments had no significant effect on egg width, eggshell weight and thickness. Similarly, Parvez et al. (2017) stated that bromocriptine had no significant effect on egg width, eggshell weight and thickness. Moreover, Banu et al. (2016) showed no significant difference in eggshell percent and shell thickness between control and bromocriptine-treated groups. The weight of an egg has a direct impact on the size and thickness of the shell (Zita et al., 2009). Lineally, AlBatshan et al. (1994) found that older hens were less efficient in absorbing calcium than younger ones. Aged laying hens at the late phase of production might have exhausted intestinal tract that limits nutrient absorption efficiency (Abdelqader et al., 2013). Furthermore, Chang-Ho et al. (2014) reported that eggshell strength gradually decreased, but eggshell thickness did not show any trends during the laying period.

Interestingly, the outcomes revealed that bromocriptine and nano-bromocriptine improved significantly egg yolk weight, width and height. This could be due to the effect of bromocriptine and its nano-form on prolactin release, as bromocriptine depressed the prolactin hormone in laying hens (Reddy et al., 2001). This could improve Follicular Stimulating Hormone (FSH) and Luteinizing Hormone (LH) function as well as ovarian tissue function. The seasonal changes in reproductive activities seen in poultry could be due to the alternative secretion of LH and prolactin, as they play stimulatory and inhibitory roles, respectively (Huang et al., 2008). Also, bromocriptine has an antioxidant effect (Lim et al., 2009), which may improve the internal egg quality and overcome the deterioration of aging (Molnár et $a l ., 2016)$. These findings were disagreed with (Banu et al., 2016; Parvez et al., 2017), as they revealed no significant effect for bromocriptine on egg quality characteristics as egg weight, egg length, egg width, albumen percent, yolk percent, shell thickness, albumen height and Haugh unite score in laying hens.

\section{Conclusion}

Either bromocriptine or nanobromocriptine could be used to improve the internal egg quality including thick albumen height, yolk weight and height during the late laying stage without any harmful effects upon external egg quality. Further studies about its impact on other performance and health parameters such as carcass quality, blood parameters and liver functions are in progress.

\section{Acknowledgment}

The research team thanks Dr. Korany A. Ali, Center of Excellence for Advanced Science, Advanced Materials and Nanotechnology Group, Applied Organic Chemistry Department, National Research Centre, Dokki, Giza 12622, Egypt and Dr. Omaima M Kandil, Center of Excellence for Embryo and Genetic Resources Conservation Bank, Department of Animal Reproduction and Artificial Insemination, Veterinary Research Division, National Research Center, Giza, Egypt for preparing the alginatebromocriptine nanocomposite.

\section{Author Contribution's}

Ahmed Dawod, Noha Osman and Hamada Mahboub: Planned and executed all experiments, dataanalysis, organization and writing of the manuscript.

Ahmed Dawod, Hanim S. Heikal and Hamada Mahboub: Designed the research plan, organized and revised writing of the manuscript. All authors read and approve the final manuscript.

\section{Funding}

This research did not receive any grant fund from any funding agency.

\section{Ethics}

The experimentation procedures and methodology of this study was done according to the guidelines of the of Helsinki and permitted via the Institutional Animal Care and Use Committee (IACUC), Faculty of Veterinary Medicine, University of Sadat City, Egypt (Ethical approval number: VUSC-017-1-19)

\section{References}

Abdelqader, A., Irshaid, R., \& Al-Fataftah, A. R. (2013). Effects of dietary probiotic inclusion on performance, eggshell quality, cecal microflora composition and tibia traits of laying hens in the late phase of production. Tropical animal health and production, 45(4), 1017-1024. doi.org/10.1007/s11250-012-0326-7

Al-Batshan, H. A., Scheideler, S. E., Black, B. L., Garlich, J. D., \& Anderson, K. E. (1994). Duodenal calcium uptake, femur ash and eggshell quality decline with age and increase following molt. Poultry science, 73(10), 1590-1596.. doi.org/10.3382/ps.0731590 
Alwell, J. J. S., Abdur-Rahman, A., \& Chukwujindu, N. S. (2017). A critical review of production factors that influence egg quality traits. age, 8, 13-18.

Banu, M. N., Rashid, M. B., Hasan, M. M., Aziz, F. B., Islam, M. R., \& Haque, M. A. (2016). Effect of antiprolactin drug and peppermint on broodiness, laying performance and egg quality in indigenous hens. Asian Journal of Medical and Biological Research, 2(4), 547-554. doi.org/10.3329/ajmbr.v2i4.30995

Bar, A., Razaphkovsky, V., \& Vax, E. (2002). Reevaluation of calcium and phosphorus requirements in aged laying hens. British poultry science, 43(2), 261-269. doi.org/10.1080/00071660120121481

Chaiseha, Y., \& El Halawani, M. E. (2005). Neuroendocrinology of the female turkey reproductive cycle. The Journal of Poultry Science, 42(2), 87-100. doi.org/10.2141/jpsa.42.87

Chang-Ho, K., Song, J. H., Lee, J. C., \& Lee, K. W. (2014). Age-related changes in egg quality of Hy-Line brown hens. International Journal of Poultry Science, 13(9), 510. doi.org/10.3923/ijps.2014.510.514

Coucke, P., Dewil, E., Decuypere, E., \& De Baerdemaeker, J. (1999). Measuring the mechanical stiffness of an eggshell using resonant frequency analysis. British Poultry Science, 40(2), 227-232. doi.org/10.1080/00071669987647

Dunn, I. C. (2013). Long Life Layer; genetic and physiological limitations to extend the laying period. In Proceedings of the 19th European Symposium on Poultry Nutrition.

Huang, Y. M., Shi, Z. D., Liu, Z., Liu, Y., \& Li, X. W. (2008). Endocrine regulations of reproductive seasonality, follicular development and incubation in Magang geese. Animal Reproduction Science, 104 (2-4), 344-358.

doi.org/10.1016/j.anireprosci.2007.02.005

Hussein, Z. A., \& Rajab, N. A. (2018). Formulation and Characterization of Bromocriptine Mesylate as Liquid Self-Nano Emulsifying Drug Delivery System. Iraqi Journal of Pharmaceutical Sciences (P93-101. ISSN-10: 1683-3597, E-ISSN: 2521-3512), doi.org/10.31351/vol27iss2pp93-101

Jaiswal, V., \& Madhav, N. V. S. (2019). Formulation Approach for bromocriptine delivery in nano form via oro- trans labio mucosal route and its performance evaluation. International Journal of Life Sciences and Review, 5, 74-81. doi.org/10.13040/IJPSR.0975-8232

Keche, Y. (2010). Bromocriptine mesylate: Food and Drug Administration approved new approach in therapy of non-insulin dependant diabetes mellitus with poor glycemic control. Journal of Pharmacy and Bioallied Sciences, 2(2), 148. doi.org/10.4103/0975-7406.67000
Lim, J. H., Kim, S. S., Boo, D. H., No, H., Kang, B. Y., Kim, E. M., ... \& Choi, H. J. (2009). Protective effect of bromocriptine against BH4-induced Cath. a cell death involving up-regulation of antioxidant enzymes. Neuroscience letters, 451(3), 185-189. doi.org/10.1016/j.neulet.2008.12.056

Molik, E., \& Blasiak, M. (2015). The Role of Melatonin and Bromocriptine in the Regulation of Prolactin Secretion in Animals-A Review. Annals of Animal Science, 15(4), 849. doi.org/10.1515/aoas-2015-0042

Molnár, A., Maertens, L., Ampe, B., Buyse, J., Kempen, I., Zoons, J., \& Delezie, E. (2016). Changes in egg quality traits during the last phase of production: Is there potential for an extended laying cycle?. British Poultry Science, 57(6), 842-847. doi.org/10.1080/00071668.2016.1209738

Nys, Y. (2001, September). Recent developments in layer nutrition for optimising shell quality. In Proc. 13th European Symposium on Poultry Nutrition. Blankenberge, Belgium (pp. 42-52). doi.org/10.1136/vr.149.19.590

Parvez, M.M., Rashid, B., Hasan, M., Mobarak, H., Roy, K,K, \& Haque, M.A. (2017). Effect of serum from laying hen and antiprolactin drug on egg production of indigenous chicken in Bangladesh. Asian Australasian Journal of Bioscience and Biotechnology, 2, 51-54

Reddy, I. J., David, C. G., \& Raju, S. S. (2007). Effect of suppression of plasma prolactin on luteinizing hormone concentration, intersequence pause days and egg production in domestic hen. Domestic animal endocrinology, 33(2), 167-175. doi.org/10.1016/j.domaniend.2006.05.002

Reddy, I. J., David, C. G., Sarma, P. V., \& Singh, K. (2001). Modulation of prolactin hormone and intersequence pause days in domestic chickens. The Veterinary Record, 149(19), 590. doi.org/10.1136/vr.149.19.590

Siddique, Y. H., Khan, W., Fatima, A., Jyoti, S., Khanam, S., Naz, F., ... \& Naqvi, A. H. (2016). Effect of bromocriptine alginate nanocomposite (BANC) on a transgenic Drosophila model of Parkinson's disease. Disease Models and Mechanisms, 9(1), 63-68. doi.org/10.1242/dmm.022145

Thiruvenkadan, A. K., Panneerselvam, S., \& Prabakaran, R. (2010). Layer breeding strategies: An overview. World's Poultry Science Journal, 66(3), 477-502. doi.org/10.1017/S0043933910000553

Van Den Brand, H., Parmentier, H. K., \& Kemp, A. B. (2004). Effects of housing system (outdoor vs cages) and age of laying hens on egg characteristics. British poultry science, $45(6), 745-752$. doi.org/10.1080/00071660400014283 
Ahmed Dawod et al. / American Journal of Pharmacology and Toxicology 2021, Volume 16: 17.24 DOI: 10.3844/ajptsp.2021.17.24

Vlčková, J., Tůmová, E., Ketta, M., Englmaierová, M., \& Chodová, D. (2018). Effect of housing system and age of laying hens on eggshell quality, microbial contamination and penetration of microorganisms into eggs. Czech Journal of Animal Science, 63(2), 51-60. https://doi.org/10.17221/77/2017-CJAS
Zita, L., Tůmová, E., \& Štolc, L. (2009). Effects of genotype, age and their interaction on egg quality in brown-egg laying hens. Acta Veterinaria Brno, 78(1), 85-91. https://actavet.vfu.cz/78/1/0085/ 